\title{
Risk factors for thyroid hormone replacement therapy after hemithyroidectomy and development of a predictive nomogram
}

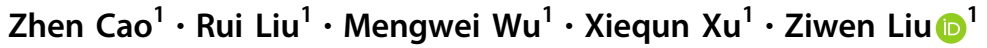 \\ Received: 28 October 2021 / Accepted: 19 December 2021 / Published online: 24 January 2022 \\ (c) The Author(s), under exclusive licence to Springer Science+Business Media, LLC, part of Springer Nature 2021
}

\begin{abstract}
Purpose Hemithyroidectomy is a valid operation to retain functional contralateral thyroid lobe that is indicated for a variety of thyroid diseases. This study aimed at determination of the risk factors for thyroid hormone replacement following hemithyroidectomy and to develop a predictive nomogram.

Methods Data of patients treated by hemithyroidectomy for benign thyroid disease between January 2015 and January 2020 were retrospectively analyzed. Baseline characteristics, surgery-related variables, and preoperative and postoperative thyroid function of patients were collected from the case records and compared between patients with postoperative euthyroidism and patients with postoperative hypothyroidism. Postoperative euthyroidism patients without thyroid hormone replacement were compared to those who developed postoperative hypothyroidism with thyroid hormone replacement. The factors associated with thyroid hormone replacement were used to construct a binomial logistic-regression model and visualized as a predictive nomogram to evaluate the risk of thyroid hormone replacement following hemithyroidectomy.

Results Of the 378 patients (74\% female) included in the study, $110(29.1 \%)$ developed postoperative hypothyroidism. Preoperative serum thyroid-stimulating hormone $(\mathrm{TSH})>2.172 \mu \mathrm{IU} / \mathrm{mL}$ was identified as an independent risk factor for postoperative hypothyroidism (odds ratio $[\mathrm{OR}]=8.02 ; 95 \%$ confidence interval $[\mathrm{CI}]: 4.87-13.20 ; P<0.001$ ). Of 110 patients with postoperative hypothyroidism, $56(50.9 \%)$ received thyroid hormone replacement. Unilateral thyroid nodule and preoperative serum TSH $>2.172 \mu \mathrm{IU} / \mathrm{mL}$ were independent predictors of postoperative thyroid hormone replacement ( $P=0.01$, and $P<0.001$, respectively). Temporary subclinical hypothyroidism occurred in 12 patients; all 12 reverted to euthyroid state without thyroid hormone replacement. The discriminative effect of the binomial regression model was proved reliable by the Hosmer-Lemeshow goodness-of-fit test $(P=0.503)$, and predictive ability of the nomogram was satisfactory with a C-index of 0.833 .

Conclusions Hypothyroidism is common after hemithyroidectomy, and almost half of the patients will need thyroid hormone replacement. Elevated preoperative serum TSH level and unilateral thyroid nodule were independent predictors of thyroid hormone replacement following hemithyroidectomy. The predictive nomogram could be a useful tool for clinical practice.
\end{abstract}

Keywords Hemithyroidectomy $\cdot$ Thyroid hormone replacement $\cdot$ Thyroid-stimulating hormone (TSH) $\cdot$ Nomogram

These authors contributed equally: Zhen Cao, Rui Liu

Xiequn $\mathrm{Xu}$

xxq75@163.com

$\triangle$ Ziwen Liu

liuziwen@pumch.cn

1 Department of General Surgery, Peking Union Medical College Hospital, Chinese Academy of Medical Sciences and Peking Union Medical College, 100730 Beijing, People's Republic of China

\section{Introduction}

Hemithyroidectomy is performed for a variety of indications, including benign and malignant thyroid disease and pathologically indeterminate nodule [1, 2]. Endogenous thyroid hormone (TH) secretion from the remained functional contralateral thyroid lobe usually fulfills the physiological need for TH [3]. However, 11-45\% of patients will develop hypothyroidism and require TH replacement [4-6].

To avoid the ill effects of hypothyroidism on the body, some surgeons start TH supplementations immediately after surgery. However, long-term TH therapy may itself cause adverse effects such as hypocalcemia, myocardial damage 
and auricular fibrillation [7-9]. Therefore, it is important to precisely identify patients who are at risk for hypothyroidism after hemithyroidectomy, then give the TH supplementation therapy.

Known risk factors for TH replacement after hemithyroidectomy include elevated preoperative serum thyroidstimulating hormone (TSH) level, presence of thyroid peroxidase antibody (TPOAb), and concomitant thyroiditis $[5,10-13]$. However, the predictive value of some other clinical factors remains unclear and controversial. The aims of this study were to (1) explore the incidence of hypothyroidism following hemithyroidectomy, (2) identify the risk factors for TH replacement after thyroid surgery, and (3) develop a binomial logistic-regression model for predicting the need for $\mathrm{TH}$ replacement after hemithyroidectomy. Surgeons can give patients preoperative counseling and postoperative guidance by using this nomogram. Based on the STROBE reporting checklists, we present this article.

\section{Materials and methods}

\section{Patient selection}

The study population was selected from 1076 patients with benign thyroid disease who underwent hemithyroidectomy between January 2015 and January 2020 at Peking Union Medical College Hospital. All thyroid surgeries were performed by the same group of surgeons at one institution. The procedure performed was confirmed from the operative note. A hemithyroidectomy was defined as complete removal of unilateral thyroid lobe and preservation of the contralateral lobe with or without isthmusectomy. The preserved isthmus was sewn using 2-0 polypropylene sutures. The types of hemithyroidectomy performed included conventional open or endoscopic procedure based on patient preference. Permission for clinical data collection was granted by the Peking Union Medical College Hospital Ethics Committee. The inclusion criteria were (1) normal preoperative serum TSH level, (2) age $\geq 18$ years at the time of surgery. The exclusion criteria were (1) preexisting hyperthyroidism or hypothyroidism $(n=47)$, (2) less than 12 months follow-up $(n=456)$, (3) receipt of TH immediately after surgery $(n=152)$, (4) contralateral thyroid lobectomy performed after hemithyroidectomy $(n=6),(5)$ history of previous thyroid surgery $(n=18)$, or (6) history of thyroid cancer, cervical lymph node cancer, or pituitary disease $(n=19)$. A total of 378 patients met these criteria.

\section{Pre- and postoperative assessment}

The electronic medical records of the selected patients were accessed and data were collected on demographic characteristics, body mass index (BMI), admission diagnosis, surgery-related variables, pathological findings, and preoperative and postoperative thyroid function. Thyroid function tests (TFT) were assessed via electrochemiluminescence immunoassay of serum TSH, TPOAb, thyroglobulin antibody ( $\mathrm{TgAb}$ ), free triiodothyronine (FT3), free thyroxine (FT4), triiodothyronine(T3), and thyroxine (T4) levels. At our institution, the reference ranges of TSH, TPOAb, TgAb, FT3, FT4, T3, and T4 are, respectively, $0.380-4.340 \mu \mathrm{IU} / \mathrm{mL}, \quad<34 \mathrm{IU} / \mathrm{mL}, \quad<115 \mathrm{IU} / \mathrm{mL}$, $1.80-4.10 \mathrm{pg} / \mathrm{mL}, 0.81-1.89 \mathrm{ng} / \mathrm{dL}, 0.66-1.92 \mathrm{ng} / \mathrm{mL}$, and $4.30-12.50 \mu \mathrm{g} / \mathrm{dL}$. Normal thyroid function was confirmed on the TFT before surgery. During the follow-up period, patients underwent thyroid function assessments at $1,3,6$, and 12 months postoperatively, then every six months after that. However, when patients complained the clinical symptoms related to thyroid dysfunction or had suspicious TFT results, TFTs were performed at any time. The presence of hypothyroidism symptoms included a puffy face, fatigue, and weight gain. The development of hypothyroidism, type of hypothyroidism, TSH level at diagnosis, the interval between operation and the development of hypothyroidism, spontaneous recovery from hypothyroidism, and the use of TH replacement were recorded.

Based on the results of postoperative TFT, patients were categorized into two groups: a postoperative euthyroid group (patients with both TSH and FT4 levels within reference ranges, $n=268$ ) and a postoperative hypothyroid group (patients with subclinical and overt hypothyroidism, $n=110$ ). Subclinical hypothyroidism was characterized by elevated serum TSH with normal FT4. Overt hypothyroidism was characterized by elevated serum TSH levels with low FT4 levels. Of the 110 postoperative hypothyroid patients, 56 patients received $\mathrm{TH}$ replacement, and 54 patients were not medicated due to no obvious discomfortable symptoms. According to the TH replacement following hemithyroidectomy, postoperative euthyroidism patients without TH replacement $(n=211)$ were compared to those who developed postoperative hypothyroidism with TH replacement $(n=56)$. Of the 54 patients without $\mathrm{TH}$ replacement, 48 patients were diagnosed with subclinical hypothyroidism and six patients were diagnosed with overt hypothyroidism. Postoperative subclinical hypothyroidism patients without $\mathrm{TH}$ replacement were of two types: those who spontaneously reverted to euthyroidism $(n=36)$ and those with persistent subclinical hypothyroidism $(n=12)$.

\section{Statistical analysis}

Categorical variables were summarized as frequencies and percentages and analyzed by the chi-square test. Continuous variables were summarized as means \pm standard deviations or medians and interquartile ranges and compared using one 
way analysis of variance (ANOVA) or the Kruskal-Wallis test. Receiver operating characteristic (ROC) curve analysis was used to identify the optimum cutoff value of preoperative serum TSH for predicting the probability of postoperative TH replacement. Binomial logistic regression was performed to evaluate the impact of each variable in risk of postoperative TH replacement. Variables associated with need for TH therapy (at the significance level of $P<$ $0.1)$ in univariate analysis were entered into multivariate analysis; the odds ratios (OR) and 95\% confidence intervals (CIs) were calculated. Two-sided $P<0.05$ was considered statistically significant. The identified variables were used to construct a visual nomogram for predicting the probability of TH replacement after surgery. The accuracy of this model was proven by the Hosmer-Lemeshow test. Internal validation of the nomogram was performed using a corrected calibration curve within 1000 bootstrap samples. Harrell concordance index (C-index) and ROC curves was used to assess prediction performance and discrimination, with C-index close to 1 indication of good predictive accuracy. Statistical analysis was carried out using SPSS 25.0 (IBM Corp., Armonk, NY, USA) and R 4.0.3 (https:// cran.r-project.org/).

\section{Results}

\section{Incidence and characteristic of hypothyroidism following hemithyroidectomy}

Of the identified 378 patients, $268(70.9 \%)$ patients had postoperative euthyroidism, $110(29.1 \%)$ developed postoperative hypothyroidism. It presents a comparison of the baseline characteristics of these two groups (Table 1). Compared to the euthyroidism group, the hypothyroidism group had significantly lower mean preoperative FT3 and FT4 level, higher mean TSH, higher proportion of patients with serum $\mathrm{TSH}>2.172 \mu \mathrm{IU} / \mathrm{mL}$ (i.e., the TSH cutoff identified by ROC analysis, area under the curve $=0.682$, 95\%CI, 0.621-0.742; sensitivity, 54.0\%; specificity, 78.5\%; Fig. 1), higher proportion of patients positive for TPOAb, and higher proportion of patients with concurrent Hashimoto thyroiditis. Patients with persistent postoperative hypothyroidism were also significantly younger than those with postoperative euthyroidism. In univariate logistic regression analysis, preoperative serum $\mathrm{TSH}>2.172 \mu \mathrm{IU} / \mathrm{mL}$ was significantly associated with likelihood of postoperative hypothyroidism (OR, 7.47; 95\% CI, 4.43 to $12.62 ; P<0.001$ ). Other variables (age at operation, preoperative TPOAb positivity, concurrent Hashimoto thyroiditis, and preoperative FT3 and FT4 levels) were not associated with postoperative hypothyroidism (Table 2). In adjusted multivariate analysis, only high preoperative TSH $(>2.172 \mu \mathrm{IU} /$
Table 1 Baseline characteristics of patients with postoperative euthyroidism and hypothyroidism

\begin{tabular}{lcll}
\hline Characteristics & $\begin{array}{l}\text { Postoperative } \\
\text { euthyroidism } \\
(n=268)\end{array}$ & $\begin{array}{l}\text { Postoperative } \\
\text { hypothyroidism } \\
(n=110)\end{array}$ & $P^{\mathrm{a}}$ \\
\hline Age at operation, y & $48.68 \pm 12.25$ & $48.61 \pm 10.91$ & 0.018 \\
BMI, kg/m 2 & $24.40 \pm 3.15$ & $24.19 \pm 3.33$ & 0.838 \\
Sex & $76(28.4)$ & $22(20.0)$ & 0.092 \\
$\quad$ Male & $192(71.6)$ & $88(80.0)$ & \\
Female & & & 0.741
\end{tabular}

Family history of

thyroid disease

$\begin{array}{lcc}\text { No } & 251(93.7) & 102(92.7) \\ \text { Yes } & 17(6.3) & 8(7.3) \\ \text { Admission diagnosis } & & \\ \text { Unilateral nodule } & 144(53.7) & 69(62.7) \\ \text { Bilateral nodules } & 124(46.3) & 41(37.3)\end{array}$

Preop TSH, $\mu \mathrm{IU} / \mathrm{mL}$

$\begin{array}{lccc}\text { Median }\left(P_{25}, P_{75}\right) & 1.38(0.98,1.93) & 2.57(1.82,3.74) & <0.001 \\ \leq 2.172 \mu \mathrm{IU} / \mathrm{mL} & 220(82.1) & 40(36.4) & <0.001 \\ >2.172 \mu \mathrm{IU} / \mathrm{mL} & 48(17.9) & 70(63.6) & \\ \text { reop FT3, pg/mL } & 3.13(2.95,3.35) & 2.98(2.86,3.28) & 0.014 \\ \text { reop FT4, ng/dL } & 1.18(1.09,1.28) & 1.14(1.05,1.26) & 0.025 \\ \text { reop T3, ng/mL } & 1.08(0.97,1.20) & 1.06(0.95,1.18) & 0.259 \\ \text { Peop T4, } \mu \mathrm{g} / \mathrm{dL} & 7.70(6.92,8.70) & 7.69(6.79,8.74) & 0.568 \\ \text { reop TgAb } & & & 0.138 \\ \text { Negative } & 239(89.2) & 92(83.6) & \\ \text { Positive } & 29(10.8) & 18(16.4) & \end{array}$

Preop TPOAb

$\begin{array}{lrr}\text { Negative } & 241(89.9) & 87(79.1) \\ \text { Positive } & 27(10.1) & 23(20.9)\end{array}$

Operation type

$(82.8)$

Thyroid lobectomy $222(82.8) \quad 86(78.2)$

Thyroid lobectomy $\quad 46(17.2) \quad 24(21.8)$ with isthmectomy

Side of lobectomy

$\begin{array}{lcc}\text { Left } & 126(47.0) & 43(39.1) \\ \text { Right } & 142(53.0) & 67(60.9) \\ \text { Hashimoto thyroiditis } & & \\ \text { Absent } & 251(93.7) & 96(87.3) \\ \text { Present } & 17(6.3) & 14(12.7)\end{array}$

Data are presented as mean \pm standard deviation, $n(\%)$, or median $\left(P_{25}, P_{75}\right)$

BMI body mass index, Preop preoperative, TSH thyroid-stimulating hormone, FT3 free triiodothyronine, FT4 free thyroxine, T3 triiodothyronine, $T 4$ thyroxine, $T g A b$ thyroglobulin antibody, $T P O A b$, thyroid peroxidase antibody

$\mathrm{mL})$ was an independent predictor of postoperative hypothyroidism $(\mathrm{OR}, 8.02 ; 95 \% \mathrm{CI}, 4.87$ to $13.20 ; P<0.001)$. The mean interval from surgery to the development of hypothyroidism was $3.47 \pm 7.79$ months (Fig. 2). While 88 
$(80 \%)$ patients developed hypothyroidism within 3 months after hemithyroidectomy, $5(4.5 \%)$ patients manifested hypothyroidism $>12$ months after surgery.

\section{Incidence and risk factors for TH replacement following hemithyroidectomy}

Of 110 postoperative hypothyroidism patients, 56 patients finally required $\mathrm{TH}$ replacement during the follow-up; thus, the incidence of $\mathrm{TH}$ replacement following hemithyroidectomy was $50.9 \%$. The association between the characteristic of postoperative euthyroidism patients without $\mathrm{TH}$ replacement and postoperative hypothyroidism patients with $\mathrm{TH}$ replacement is shown in Table 3. Postoperative hypothyroidism patients with $\mathrm{TH}$ replacement were significantly younger than those who developed

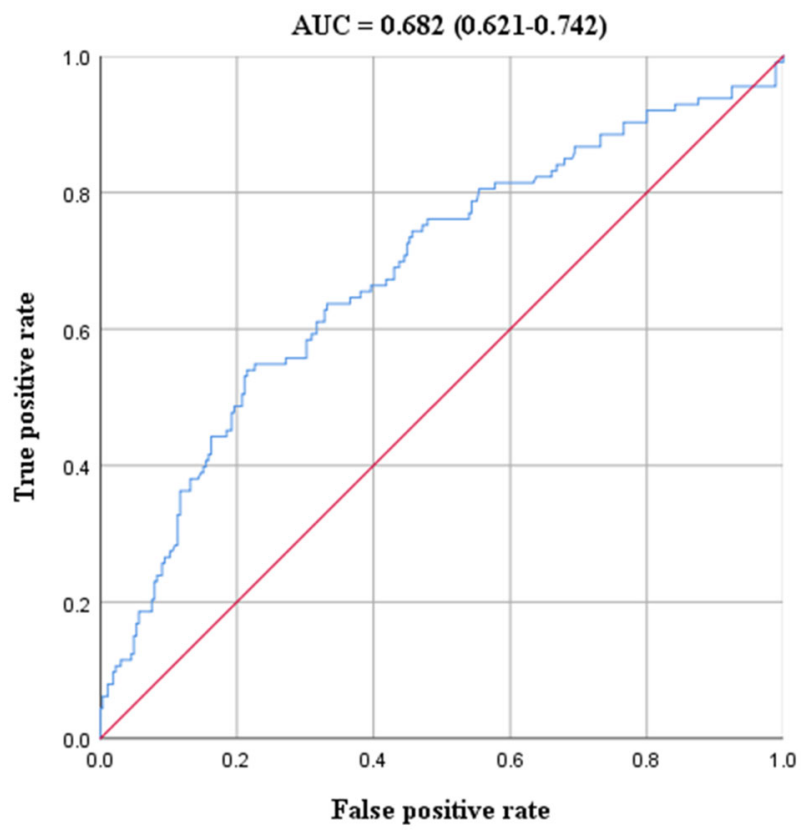

Fig. 1 The ROC curve of TSH cutoff value. The area under the ROC curve (AUC) is $0.682,95 \% \mathrm{CI}, 0.621-0.742$. ROC receiver operating characteristic, AUC area under curve postoperative euthyroidism without $\mathrm{TH}$ replacement. The TH replacement group had a higher proportion of females, patients with unilateral thyroid nodule, patients with preoperative serum TSH levels $>2.172 \mu \mathrm{IU} / \mathrm{mL}$, with right sided lobectomy, and positive for $\mathrm{TgAb}$ and TPOAb. The preoperative serum TSH was significantly higher and serum FT3, FT4, and T4 significantly lower in patients who received $\mathrm{TH}$ replacement than in those who did not receive TH replacement (all $P<0.05$ ).

It presents the results of univariate and multivariate analyses (Table 4). In univariate analysis, the factors associated with $\mathrm{TH}$ replacement included admission diagnosis of unilateral thyroid nodule $(P=0.02)$, Female $(P=0.03)$, preoperative serum TSH $>2.172 \mu \mathrm{IU} / \mathrm{mL}(P<0.001)$, low preoperative FT3, FT4 and T4 $(P=0.04, P=0.01, P=$ 0.03 , respectively), preoperative $\mathrm{TgAb}$ and TPOAb positivity $(P=0.09, P=0.01$, respectively), and right-side lobectomy $(P=0.07)$ were found to be linked with $\mathrm{TH}$ replacement following HT. Age at operation, and preoperative T3, thyroid lobectomy with isthmusectomy and present of Hashimoto thyroiditis were not associated with TH replacement $(P=0.18, P=0.16, P=0.11, P=0.30$, respectively). In adjusted multivariate analysis, only two variables were independent predictors of $\mathrm{TH}$ replacement,

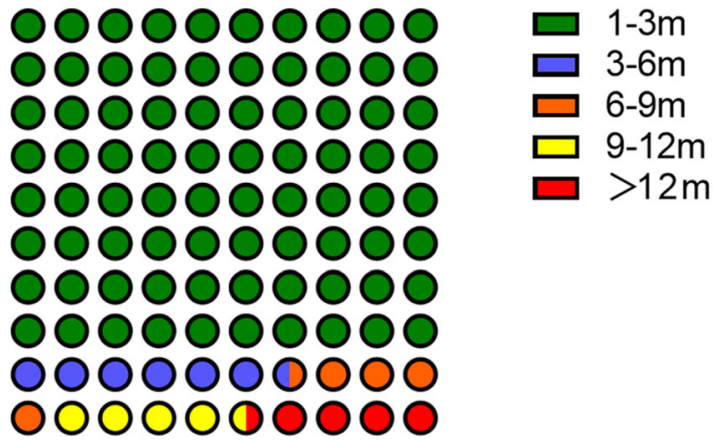

\section{Total postoperative hypothyroidism $=110$}

Fig. 2 Time interval from hemithyroidectomy to the development of hypothyroidism
Table 2 Univariate and multivariate analysis of risk factors for postoperative hypothyroidism

\begin{tabular}{llllll}
\hline Variable & Univariate & & & Multivariate & \\
\cline { 2 - 3 } & OR $(95 \% \mathrm{CI})$ & $P$ & & OR (95\% CI) & $P$ \\
\hline Age at operation & $1.00(0.98-1.03)$ & 0.77 & & \\
Preop TSH $>2.172 \mu \mathrm{IU} / \mathrm{mL}$ & $7.47(4.43-12.62)$ & $<0.001$ & & $8.02(4.87-13.20)$ & $<0.001$ \\
Lower preop FT3 & $0.59(1.20-2.45)$ & 0.61 & & \\
Lower preop FT4 & $0.41(0.07-2.35)$ & & 0.32 & & \\
Preop TPOAb positivity & $1.32(0.60-2.89)$ & & 0.49 & & \\
Hashimoto thyroiditis & $1.07(0.40-2.82)$ & & 0.90 & & \\
\hline
\end{tabular}

OR odds ratio, $C I$ confidence interval, Preop preoperative, TSH thyroid-stimulating hormone, FT3 free triiodothyronine, FT4 free thyroxine, TPOAb thyroid peroxidase antibody 
Table 3 Characteristics of postoperative euthyroidism patients without $\mathrm{TH}$ replacement and postoperative hypothyroidism patients with $\mathrm{TH}$ replacement

\begin{tabular}{|c|c|c|c|}
\hline Characteristics & $\begin{array}{l}\text { Postoperative- } \\
\text { euthyroidism } \\
\text { patients without } \\
\text { TH replacement } \\
(n=211)\end{array}$ & $\begin{array}{l}\text { Postoperative- } \\
\text { hypothyroidism } \\
\text { patients with TH } \\
\text { replacement } \\
(n=56)\end{array}$ & $P^{\mathrm{a}}$ \\
\hline Age at operation, $y$ & $49.59 \pm 12.39$ & $47.13 \pm 9.80$ & 0.006 \\
\hline BMI, $\mathrm{kg} / \mathrm{m}^{2}$ & $24.61 \pm 3.10$ & $24.29 \pm 3.61$ & 0.264 \\
\hline Sex & & & 0.024 \\
\hline Male & $66(31.3)$ & $9(16.1)$ & \\
\hline Female & $145(68.7)$ & 47 (83.9) & \\
\hline $\begin{array}{l}\text { Family history of } \\
\text { thyroid disease }\end{array}$ & & & 0.298 \\
\hline No & $200(94.8)$ & $51(91.1)$ & \\
\hline Yes & $11(5.2)$ & $5(8.9)$ & \\
\hline Admission diagnosis & & & 0.022 \\
\hline Unilateral nodule & $107(50.7)$ & $38(67.9)$ & \\
\hline Bilateral nodules & $104(49.3)$ & $18(32.1)$ & \\
\hline \multicolumn{4}{|l|}{ Preop TSH, $\mu \mathrm{IU} / \mathrm{mL}$} \\
\hline Median $\left(P_{25}, P_{75}\right)$ & $1.29(0.95,1.79)$ & $2.87(1.84,3.90)$ & $<0.001$ \\
\hline$\leq 2.172 \mu \mathrm{IU} / \mathrm{mL}$ & $184(87.2)$ & $17(30.4)$ & $<0.001$ \\
\hline$>2.172 \mu \mathrm{IU} / \mathrm{mL}$ & $27(12.8)$ & $39(69.6)$ & \\
\hline Preop FT3, g/mL & $3.13(2.94,3.32)$ & $2.97(2.85,3.26)$ & 0.015 \\
\hline Pre FT4, ng/dL & $1.18(1.09,1.29)$ & $1.10(1.03,1.26)$ & 0.006 \\
\hline Preop T3, ng/mL & $1.08(0.97,1.21)$ & $1.05(0.96,1.18)$ & 0.265 \\
\hline Preop T4, $\mu \mathrm{g} / \mathrm{dL}$ & $7.70(6.99,8.80)$ & $7.20(6.33,8.38)$ & 0.044 \\
\hline Preop $\mathrm{TgAb}$ & & & 0.038 \\
\hline Negative & $188(89.1)$ & $44(78.6)$ & \\
\hline Positive & $23(10.9)$ & $12(21.4)$ & \\
\hline Preop TPOAb & & & 0.001 \\
\hline Negative & $192(91.0)$ & $42(75.0)$ & \\
\hline Positive & $19(9.0)$ & $14(25.0)$ & \\
\hline Operation type & & & 0.103 \\
\hline Thyroid lobectomy & $181(85.8)$ & $43(76.8)$ & \\
\hline $\begin{array}{l}\text { Thyroid lobectomy } \\
\text { with } \\
\text { isthmusectomy }\end{array}$ & $30(14.2)$ & $13(23.2)$ & \\
\hline Side of lobectomy & & & 0.041 \\
\hline Left & $100(47.4)$ & $18(32.1)$ & \\
\hline Right & $111(52.6)$ & $38(67.9)$ & \\
\hline $\begin{array}{l}\text { Hashimoto } \\
\text { thyroiditis }\end{array}$ & & & 0.134 \\
\hline Absent & $200(94.8)$ & $50(89.3)$ & \\
\hline Present & $11(5.2)$ & $6(10.7)$ & \\
\hline
\end{tabular}

Data are presented as mean \pm standard deviation, $n(\%)$, or median $\left(P_{25}, P_{75}\right)$

$B M I$ body mass index, Preop preoperative, TSH thyroid-stimulating hormone, FT3 free triiodothyronine, FT4 free thyroxine, T3 triiodothyronine, $T 4$ thyroxine, $T g A b$ thyroglobulin antibody, $T P O A b$ thyroid peroxidase antibody i.e., admission diagnosis of unilateral thyroid nodule $(\mathrm{OR}=$ $2.81 ; 95 \% \mathrm{CI}: 1.26$ to $6.26, P=0.01)$ and preoperative serum $\quad \mathrm{TSH}>2.172 \mu \mathrm{IU} / \mathrm{mL} \quad(\mathrm{OR}, 17.94 ; \quad 95 \% \quad \mathrm{CI}$, 7.97-40.39; $P<0.001$ ).

Some patients with subclinical hypothyroidism spontaneously reverted to euthyroidism without TH replacement. Hence, patients with subclinical hypothyroidism who did not receive TH replacement were divided into a persistent subclinical hypothyroidism group $(n=36)$ and a reverted to euthyroidism group $(n=12)$. Table 5 presents a comparison of these two groups. Significant predictors of reversion to euthyroidism after developing subclinical hypothyroidism were not identified.

\section{Nomogram development and validation}

Based on the abovementioned nine factors enrolled into multivariate analysis, we built a logistic regression model and visualized it into a nomogram. However, the length of three factors including preoperative FT3, TgAb and side of lobectomy were too short, and when excluded, the predictive ability of the model didn't decline at a significantly level (C-index was 0.8334 before excluded and 0.8333 after). Therefore, a binomial logistic-regression model was built using six risk factors identified by univariate and multivariate analysis: i.e., sex, admission diagnosis, preoperative TSH, FT4 and T4 levels, and TPOAb positivity (Fig. 3A), and visualized as a nomogram for predicting the probability of $\mathrm{TH}$ replacement after hemithyroidectomy. The risk of medication probability predicted by this nomogram model ranged from 0.010 to 0.999 . The consistency of the model was confirmed by the Hosmer-Lemeshow goodness-of-fit test $(\chi 2=7.313$, df $=8, P=0.503)$. The calibration curves showed a largely perfect agreement between nomogrampredicted outcomes and actual outcomes (Fig. 3B). The model performed well, with Harrel's C-index of 0.833 and the area under curve (AUC) was 0.833 (95\%CI, 0.768-0.898) (Fig. 3C). Nomograms show the likelihood of TH replacement after hemithyroidectomy as a percentage, and we assigned a Youden-derived cutoff value to the nomogram. The optimal cutoff value was 39.371 (sensitivity, $73.2 \%$; specificity, $85.8 \%$; negative predictive value, $92.3 \%$; positive predictive value, $57.7 \%$ ). For clinical convenience, we uploaded an online-program using the "DynNom" package of R (https://cran.r-project. org/web/packages/DynNom/index.html); it is available at https://liuruisurgeon.shinyapps.io/TH_replacement/. After determining the parameters, the probability of $\mathrm{TH}$ replacement can be obtained by clicking the "predict" button (Fig. 4). 
Table 4 Univariate and multivariate analyses of the risk factors for TH replacement following hemithyroidectomy

\begin{tabular}{|c|c|c|c|c|}
\hline \multirow[t]{2}{*}{ Variable } & \multicolumn{2}{|l|}{ Univariate } & \multicolumn{2}{|l|}{ Multivariate } \\
\hline & OR $(95 \% \mathrm{CI})$ & $P$ & OR $(95 \% \mathrm{CI})$ & $P$ \\
\hline Age at operation & $0.98(0.96-1.00)$ & 0.18 & & \\
\hline Female & $2.38(1.10-5.14)$ & 0.03 & $1.83(0.63-5.32)$ & 0.27 \\
\hline Unilateral thyroid nodule & $2.05(1.10-3.82)$ & 0.02 & $2.81(1.26-6.26)$ & 0.01 \\
\hline Preop TSH $>2.172 \mu \mathrm{IU} / \mathrm{mL}$ & $17.04(8.41-34.54)$ & $<0.001$ & $17.94(7.97-40.39)$ & $<0.001$ \\
\hline Lower preop FT3 & $0.37(0.14-0.94)$ & 0.04 & $1.22(0.35-4.27)$ & 0.76 \\
\hline Lower preop FT4 & $0.04(0.01-0.31)$ & 0.01 & $3.01(0.14-65.7)$ & 0.48 \\
\hline Lower preop T3 & $0.27(0.04-1.67)$ & 0.16 & & \\
\hline Lower preop T4 & $0.79(0.64-0.98)$ & 0.03 & $0.77(0.58-1.03)$ & 0.77 \\
\hline Preop $\operatorname{TgAb}$ positivity & $1.99(0.91-4.40)$ & 0.09 & $1.87(0.58-6.08)$ & 0.30 \\
\hline Preop TPOAb positivity & $3.06(1.40-6.66)$ & 0.01 & $1.28(0.42-3.96)$ & 0.67 \\
\hline Thyroid lobectomy with isthmusectomy & $1.83(0.88-3.78)$ & 0.11 & & \\
\hline Right-side lobectomy & $1.75(0.95-3.25)$ & 0.07 & $1.69(0.78-3.66)$ & 0.19 \\
\hline Present of Hashimoto thyroiditis & $1.78(0.59-5.36)$ & 0.30 & & \\
\hline
\end{tabular}

Preop preoperative, TSH thyroid-stimulating hormone, FT3 free triiodothyronine, FT4 free thyroxine, T4 thyroxine, $T g A b$ thyroglobulin antibody, $T P O A b$ thyroid peroxidase antibody, $O R$ odds ratio, $C I$ confidence interval

\section{Discussion}

The overall incidence of hypothyroidism after surgery was $29.1 \%$ in our cohort, and almost half of them received postoperative TH replacement, which was consistent with earlier studies varied from 11 to $45 \%[5,6,10,11,14]$. The mean interval from surgery to the development of hypothyroidism was 3.47 months in our study. Most cases of hypothyroidism occurred in the early follow-up period; $80 \%$ of patients had developed hypothyroidism within 3 months, and about 90\% within 9 months. Thus, the findings suggested that monitoring period of thyroid function for at least 9 months after hemithyroidectomy would ensure that at least $90 \%$ of hypothyroidism cases were detected. In a retrospective analysis of 405 patients undergoing hemithyroidectomy, Ahn et al. [4] reported similar results. In our study, nearly $5 \%$ of patients were diagnosed with postoperative hypothyroidism beyond 12 months. Therefore, we recommended prolonged follow-up of thyroid function, particularly for patients with high risk of hypothyroidism.

Multiple prior studies have found that elevated preoperative serum TSH, presence of TPOAb, and concomitant thyroiditis are predictors of need for postoperative $\mathrm{TH}$ replacement $[2,5,10,11,15,16]$. In our study, besides elevated preoperative serum TSH, admission diagnosis of unilateral thyroid nodule was also found to be predictor of TH replacement therapy. Consistent with previous studies, preoperative TSH level had the strongest predictive value (highest odds ratio) [16]. In our opinion, higher preoperative TSH levels indicated lower reserved thyroid function and, therefore, greater likelihood of developing hypothyroidism after surgery. ROC analysis identified preoperative TSH $2.172 \mu \mathrm{IU} / \mathrm{mL}$ as the best cutoff value for identifying patients at high risk of developing postoperative hypothyroidism. Hypothyroidism developed in $63.6 \%$ of patients with $\mathrm{TSH}>2.172 \mu \mathrm{IU} / \mathrm{mL}$ vs. $36.4 \%$ of patients with $\mathrm{TSH} \leq 2.172 \mu \mathrm{IU} / \mathrm{mL}(P<0.001)$. The cut-off value identified in previous studies ranged from 1.4 to $2.5 \mathrm{mIU} / \mathrm{L}$ $[16,17]$.

A surprising finding was that unilateral thyroid nodule was an independent predictor of postoperative $\mathrm{TH}$ replacement. This finding is different from what surgeons usually think and has not been accurately reported before. In a study of 150 patients undergoing hemithyroidectomy, Lang et al. [18] found that fewer number of ipsilateral nodules was associated with increased risk for hypothyroidism after surgery. This is, to some extent, similar to our finding. We boldly wondered if there were bilateral nodules that increased compensatory thyroid function. Contralateral thyroid nodules after hemithyroidectomy boosted thyroid function. Based on more clinical studies, further research is needed to clarify the link between unilateral thyroid nodule and postoperative hypothyroidism.

In this study, we separately evaluated patients with subclinical hypothyroidism; this subgroup has not been adequately assessed in previous studies [19, 20]. In our sample, $25 \%$ of patients with subclinical hypothyroidism following hemithyroidectomy reverted to euthyroidism without TH replacement. However, statistically significant predictors of reversion to euthyroid state were not identified, probably because our sample size was small. 
Table 5 Characteristics of patients with subclinical hypothyroidism not receiving $\mathrm{TH}$ replacement after surgery

\begin{tabular}{llll}
\hline Characteristics & $\begin{array}{l}\text { Persistent } \\
\text { subclinical } \\
\text { hypothyroidism } \\
(n=36)\end{array}$ & $\begin{array}{l}\text { Spontaneously } \\
\text { reverted to } \\
\text { euthyroidism } \\
(n=12)\end{array}$ & $P$ \\
\hline Age at operation, y & $50.33 \pm 11.90$ & $49.42 \pm 14.65$ & 0.464 \\
BMI, kg/m ${ }^{2}$ & $24.04 \pm 2.85$ & $24.39 \pm 3.21$ & 0.451 \\
Sex & $10(27.8)$ & $1(8.3)$ & 0.322 \\
$\quad$ Male & $26(72.2)$ & $11(91.7)$ & \\
Female & &
\end{tabular}

Family history of thyroid disease

$\begin{array}{clll}\text { No } & 34(94.4) & 11(91.7) & \\ \text { Yes } & 2(5.6) & 1(8.3) & >0.99 \\ \text { Admission diagnosis } & & & \\ \text { Unilateral nodule } & 21(58.3) & 7(58.3) & \\ \text { Bilateral nodules } & 15(41.7) & 5(41.7) & \end{array}$

Preop TSH level, $\mu \mathrm{IU} / \mathrm{mL}$

\begin{tabular}{|c|c|c|c|}
\hline$\leq 2.172 \mu \mathrm{IU} / \mathrm{mL}$ & $18(50.0)$ & $6(50.0)$ & \\
\hline$>2.172 \mu \mathrm{IU} / \mathrm{mL}$ & $18(50.0)$ & $6(50.0)$ & \\
\hline Preop FT3, pg/mL & $3.08(2.93,3.42)$ & $2.91(2.75,3.12)$ & 0.067 \\
\hline reop FT4, ng/dL & $1.19(1.12,1.27)$ & $1.22(1.07,1.29)$ & 0.153 \\
\hline Preop T3, ng/mL & $1.13(0.94,1.20)$ & $1.00(0.98,1.08)$ & 0.721 \\
\hline Preop T4, $\mu \mathrm{g} / \mathrm{dL}$ & $8.21(7.47,8.90)$ & $7.84(7.15,9.30)$ & 0.592 \\
\hline Preop $\mathrm{TgAb}$ & & & 0.546 \\
\hline Negative & $34(94.4)$ & $10(83.3)$ & \\
\hline Positive & $2(5.6)$ & $2(16.7)$ & \\
\hline Preop TPOAb & & & $>0.99$ \\
\hline Negative & $32(88.9)$ & $10(83.3)$ & \\
\hline Positive & $4(11.1)$ & $2(16.7)$ & \\
\hline Operation type & & & 0.655 \\
\hline Thyroid lobectomy & $29(80.6)$ & $11(91.7)$ & \\
\hline $\begin{array}{l}\text { Thyroid lobectomy } \\
\text { with } \\
\text { isthmusectomy }\end{array}$ & 7 (19.4) & $1(8.3)$ & \\
\hline Side of lobectomy & & & $>0.99$ \\
\hline Left & $15(41.7)$ & $5(41.7)$ & \\
\hline Right & $21(58.3)$ & $7(58.3)$ & \\
\hline Hashimoto thyroiditis & & & $>0.99$ \\
\hline Absent & $32(88.9)$ & $11(91.7)$ & \\
\hline Present & $4(11.1)$ & $1(8.3)$ & \\
\hline
\end{tabular}

Data are presented as mean \pm standard deviation, $n(\%)$, or median $\left(P_{25}, P_{75}\right)$

BMI body mass index, Preop preoperative, TSH thyroid-stimulating hormone, FT3 free triiodothyronine, FT4 free thyroxine, T3 triiodothyronine, $T 4$ thyroxine, $T g A b$ thyroglobulin antibody, TPOAb thyroid peroxidase antibody

Buehler et al. [10] found that nearly half of their patients with subclinical hypothyroidism recovered to normal thyroid function within a 5-year follow-up period. The larger proportion identified probably due to the longer follow-up and included observation of thyroid cancer patients. These findings suggested that all patients who develop postoperative subclinical hypothyroidism could not need immediate $\mathrm{TH}$ replacement.

A binomial logistic-regression model was built using the variables significantly associated with $\mathrm{TH}$ replacement, and visualized as a nomogram for predicting the probability of TH replacement after surgery. Multiple previous studies have proved that the nomogram has better predictive performance [21-23]. To our knowledge, this is the first report of a nomogram for predicting benign thyroid disease. Our model was shown to be reliable, with a good C-index. According to previous study, an AUC value greater than 0.7 has superior accuracy, indicating good discrimination. With an optimal cutoff value of 39.371 , the nomogram yielded $73.2 \%$ sensitivity, $85.8 \%$ specificity. Therefore, patients with a total point value of more than 39.371 are at high risk for TH replacement. However, close observation with TFT and follow-up are recommended for patients with a total point value of less than 39.371 . Thus, the ability to predict TH replacement can be useful considering the side effects of TH supplementation and, currently, the difficulty in following up patients due to the COVID-19 pandemic [24].

Several studies reported that an elevated TSH level were associated with a higher incidence of thyroid carcinoma and advanced diseases. Therefore, some surgeons believed that thyroid cancer patients should be supplemented with levothyroxine to suppress TSH to prevent possible tumor recurrence or progression. They focused on patients with thyroid carcinoma to explore the risk factors and determined the optimum suppressed TSH level to improve the prognosis [25-27]. Nevertheless, some surgeons considered that there was little evidence to guide TSH suppression therapy in low-risk patients who have undergone lobectomy $[4,17]$. They evaluated the risk factors of hypothyroidism following hemithyroidectomy and focused on patients with benign thyroid disease and thyroid carcinoma. In our study, the identified 378 patients underwent hemithyroidectomy for benign thyroid disease. Since TH replacement were often given in patients with postoperative hypothyroidism following hemithyroidectomy rather than suppressive therapy, we merely included patients with benign thyroid disease to analyze and excluded ones with thyroid cancer.

Our study has several limitations. First, although unilateral thyroid nodule was identified as an independent risk factor for $\mathrm{TH}$ replacement, we did not measure the number and volume of thyroid nodules by ultrasonography. The reason why presence of the contralateral thyroid nodules relatively acts as a protective factor remains unexplained. Second, we did not quantitatively assess chronic lymphocytic infiltration by histopathology which has been previously identified as a potential predictor of $\mathrm{TH}$ 
Fig. 3 A binomial logisticregression model $(\mathbf{A})$

Nomogram for predicting the probability of thyroid hormone replacement following hemithyroidectomy, (B) calibration curves of the nomogram. The $45^{\circ}$ dashed line represents the ideal predictions (ideal line). The solid line is a bias-corrected curve. The dotted line is the nomogram probability and $(\mathbf{C})$ the ROC curve of nomograms for predicting the probability of $\mathrm{TH}$ replacement. The area under the ROC curve (AUC) is $0.833,95 \% \mathrm{CI}$ 0.768-0.898. ROC receiver operating characteristic, AUC area under curve
A

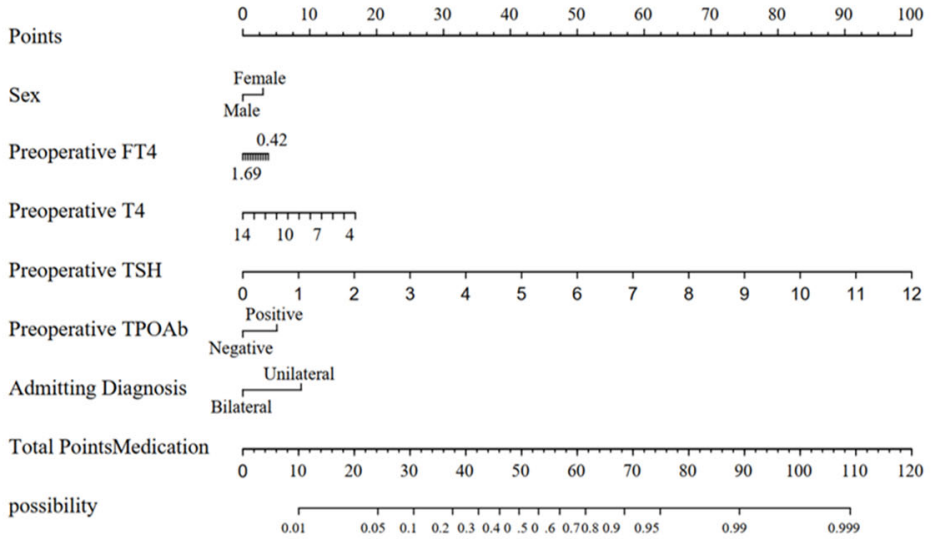

B

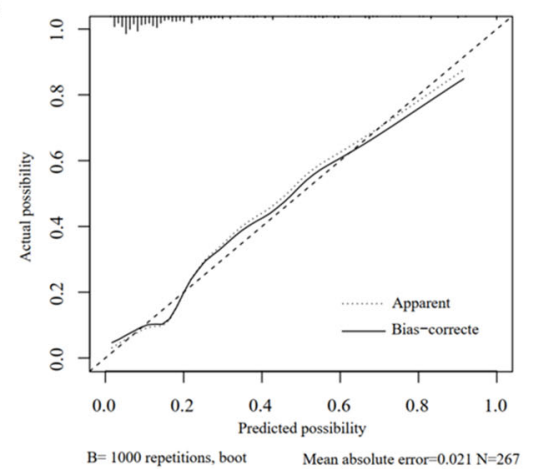

C

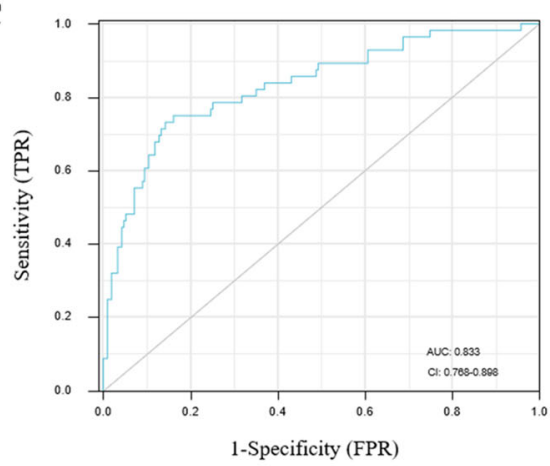

Dynamic Nomogram

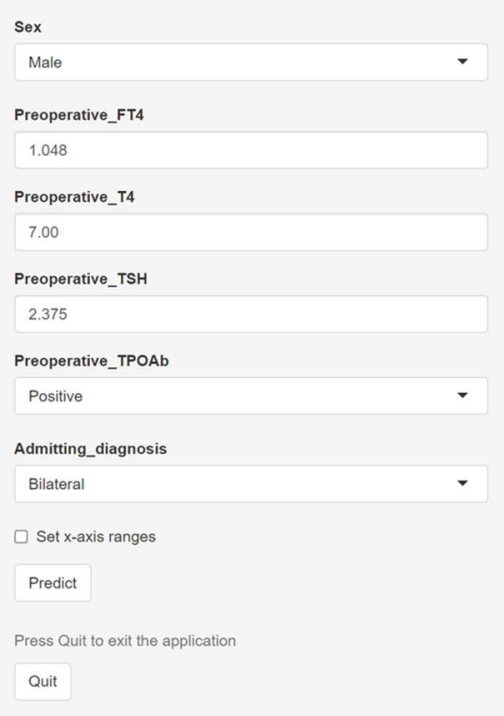

\begin{tabular}{|c|c|c|c|c|c|c|c|c|}
\hline \multicolumn{2}{|c|}{ Graphical Summary } & Numerical Summary & Model Summar & & & & & \\
\hline $\begin{array}{c}=z=z= \pm= \\
\text { Sex }\end{array}$ & Preoperativ & 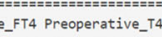 & 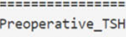 & operative_TPOAb & Admitting_diagnosis & Prediction & Lower. bound & $\begin{array}{l}\text { =ezezezez } \\
\text { Upper.bounc }\end{array}$ \\
\hline 1 Female & 1.359 & 9.100 & 1.484 & Negative & Bilateral & 0.054 & 0.022 & 0.127 \\
\hline $2 \quad$ Male & 1.234 & 10.750 & 2.898 & Negative & Unilateral & 0.250 & 0.088 & 0.536 \\
\hline 3 Female & 0.980 & 5.780 & 2.870 & Negative & Unilateral & 0.580 & 0.400 & 0.740 \\
\hline $4 \quad$ Male & 1.328 & 6.260 & 4.217 & Negative & Unilateral & 0.734 & 0.421 & 0.913 \\
\hline 5 Female & 1.092 & 8.280 & 1.444 & Negative & Unilateral & 0.166 & 0.098 & 0.268 \\
\hline 6 Male & $1.32 \theta$ & 6.260 & 4.217 & Negative & Unilateral & 0.734 & 0.421 & 0.913 \\
\hline 7 Female & 1.249 & 8.850 & 0.747 & Negative & Unilateral & 0.078 & 0.038 & 0.153 \\
\hline 8 Female & 1.179 & 8.750 & 0.972 & Negative & Unilateral & 0.100 & 0.052 & 0.181 \\
\hline 9 Female & 1.062 & 7.640 & 1.749 & Negative & Bilateral & 0.100 & 0.050 & 0.190 \\
\hline $10 \mathrm{Male}$ & 1.048 & 7 & 2.375 & Positive & Bilateral & 0.229 & 0.069 & 0.545 \\
\hline
\end{tabular}

Fig. 4 The online program for predicting the probability of thyroid hormone replacement following hemithyroidectomy is available at https:// liuruisurgeon.shinyapps.io/TH_replacement/

replacement. Third, this was a retrospective single-center study, and therefore information bias and selection bias cannot be ruled out. Fourth, external validation of the logistic model was not performed. Further studies are needed for confirmation.

\section{Conclusion}

In summary, approximately one-third of patients undergoing hemithyroidectomy may develop hypothyroidism, and of these almost half will require TH replacement. 
Elevated preoperative serum TSH level and admission diagnosis of unilateral thyroid nodule appear to be independent predictors of need for TH replacement following hemithyroidectomy. The constructed visual nomogram is a simple and objective tool that can predict the probability of need for TH replacement following hemithyroidectomy and help identify patients who need close follow-up after thyroid surgery.

\section{Data availability}

The datasets in this study are available from the corresponding author on reasonable request.

Author contributions (I) Conception and design: Z.C., X.X.; (II) Administrative support: X.X., Z.L.; (III) Provision of study materials or patients: X.X., X.L., Z.L.; (IV) Collection and assembly of data: Z.C., R.L., M.W.; (V) Data analysis and interpretation: Z.C., R.L., M.W.; (VI) Manuscript writing: All authors; (VII) Final approval of manuscript: All authors.

Funding This work was supported by the National Nature Science Foundation of China [Nos. 32071436, 81572459] for Xiequn Xu and Ziwen Liu, General Program of Beijing Nature Science Foundation [Nos. 7202164] for Ziwen Liu.

\section{Compliance with ethical standards}

Conflict of interest The authors declare no competing interests.

Ethical approval The study was conducted in accordance with the Declaration of Helsinki (as revised in 2013). The study was approved by the Ethical Committee of the Peking Union Medical College Hospital and individual consent for this retrospective analysis was waived. All the procedures being performed were part of the routine care.

Publisher's note Springer Nature remains neutral with regard to jurisdictional claims in published maps and institutional affiliations.

\section{References}

1. T.E. Angell, C.M. Vyas, J.A. Barletta, E.S. Cibas, N.L. Cho, G.M. Doherty, A.A. Gawande, B.E. Howitt, J.F. Krane, E. Marqusee, K.C. Strickland, E.K. Alexander, F.D. Moore Jr, M.A. Nehs, Reasons associated with total thyroidectomy as initial surgical management of an indeterminate thyroid nodule. Ann. Surg. Oncol. 25, 1410-1417 (2018)

2. V. Beisa, D. Kazanavicius, A. Skrebunas, G. Simutis, J. Ivaska, K. Strupas, Prospective analysis of risk for hypothyroidism after hemithyroidectomy. Int. J. Endocrinol. 2015, 313971 (2015)

3. T.K. Ha, D.W. Kim, H.K. Park, J.W. Baek, Y.J. Lee, Y.M. Park, H. Kim do, S.J. Jung, K.J. Ahn, The effect of levothyroxine discontinuation timing on postoperative hypothyroidism after hemithyroidectomy for papillary thyroid microcarcinoma. Int. J. Endocrinol. 2016, 3240727 (2016)

4. D. Ahn, J.H. Sohn, J.H. Jeon, Hypothyroidism following hemithyroidectomy: incidence, risk factors, and clinical characteristics. J. Clin. Endocrinol. Metab. 101, 1429-1436 (2016)
5. S. Park, M.J. Jeon, E. Song, H.S. Oh, M. Kim, H. Kwon, T.Y. Kim, S.J. Hong, Y.K. Shong, W.B. Kim, T.Y. Sung, W.G. Kim, Clinical features of early and late postoperative hypothyroidism after lobectomy. J. Clin. Endocrinol. Metab. 102, 1317-1324 (2017)

6. A.K. Al-Shalhoub, S. Al-Dhahri, Risk factors of posthemithyroidectomy hypothyroidism. Saudi J. Med Med Sci. 5, 45-48 (2017)

7. H. Deshmukh, M. Papageorgiou, M. Aye, J. England, M. Abdalla, T. Sathyapalan, Hyperthyroidism and bone mineral density: dissecting the causal association with Mendelian randomization analysis. Clin. Endocrinol. (Oxf.) 94, 119-127 (2021)

8. J. Munoz-Ortiz, M.C. Sierra-Cote, E. Zapata-Bravo, L. Valenzuela-Vallejo, M.A. Marin-Noriega, P. Uribe-Reina, J.P. TerrerosDorado, M. Gomez-Suarez, K. Arteaga-Rivera, A. de-la-Torre, Prevalence of hyperthyroidism, hypothyroidism, and euthyroidism in thyroid eye disease: a systematic review of the literature. Syst. Rev. 9, 201 (2020)

9. B.H. Yoon, Y. Lee, H.J. Oh, S.H. Kim, Y.K. Lee, Influence of thyroid-stimulating hormone suppression therapy on bone mineral density in patients with differentiated thyroid cancer: a metaanalysis. J. Bone Metab. 26, 51-60 (2019)

10. L.A. Buehler, N.Z. Madhun, J. Bena, C. Nasr, J. Scharpf, K. Zhou, Hormonal outcomes following hemithyroidectomy. Otolaryngol. Head. Neck Surg. 164, 1011-1018 (2021)

11. D. Ahn, G.J. Lee, J.H. Sohn, Levothyroxine supplementation following hemithyroidectomy: incidence, risk factors, and characteristics. Ann. Surg. Oncol. 26, 4405-4413 (2019)

12. J. Chen, S. Hou, X. Li, J. Yang, Management of subclinical and overt hypothyroidism following hemithyroidectomy in children and adolescents: a pilot study. Front Pediatr. 7, 396 (2019)

13. E. Kandil, B. Krishnan, S.I. Noureldine, L. Yao, R.P. Tufano, Hemithyroidectomy: a meta-analysis of postoperative need for hormone replacement and complications. ORL J. Otorhinolaryngol. Relat. Spec. 75, 6-17 (2013)

14. H. Verloop, M. Louwerens, J.W. Schoones, J. Kievit, J.W. Smit, O.M. Dekkers, Risk of hypothyroidism following hemithyroidectomy: systematic review and meta-analysis of prognostic studies. J. Clin. Endocrinol. Metab. 97, 2243-2255 (2012)

15. C.R. McHenry, S.J. Slusarczyk, Hypothyroidisim following hemithyroidectomy: incidence, risk factors, and management. Surgery 128, 994-998 (2000)

16. M. Said, V. Chiu, P.I. Haigh, Hypothyroidism after hemithyroidectomy. World J. Surg. 37, 2839-2844 (2013)

17. D.Y. Lee, J. Seok, W.J. Jeong, S.H. Ahn, Prediction of thyroid hormone supplementation after thyroid lobectomy. J. Surg. Res 193, 273-278 (2015)

18. B.H. Lang, C.K.H. Wong, K.P. Wong, K.K. Chu, T.W.H. Shek, Effect of thyroid remnant volume on the risk of hypothyroidism after hemithyroidectomy: a prospective study. Ann. Surg. Oncol. 24, 1525-1532 (2017)

19. A. Johner, O.L. Griffith, B. Walker, L. Wood, H. Piper, G. Wilkins, C. Baliski, S.J. Jones, S.M. Wiseman, Detection and management of hypothyroidism following thyroid lobectomy: evaluation of a clinical algorithm. Ann. Surg. Oncol. 18, 2548-2554 (2011)

20. P. Ng, C. Ho, W.B. Tan, K.Y. Ngiam, C.M. Lim, K.S. Thomas Loh, M.E. Nga, R. Parameswaran, Predictors of thyroxine replacement following hemithyroidectomy in a south east Asian cohort. Head. Neck 41, 1463-1467 (2019)

21. R. Zhang, M. Xu, X. Liu, M. Wang, Q. Jia, S. Wang, X. Zheng, X. He, C. Huang, Y. Fan, H. Wu, K. Xu, D. Li, Z. Meng, Establishment and validation of a nomogram model for predicting the survival probability of differentiated thyroid carcinoma patients: a comparison with the eighth edition AJCC cancer staging system. Endocrine 74, 108-119 (2021)

22. L. Chen, Y. Wang, K. Zhao, Y. Wang, X. He, Postoperative nomogram for predicting cancer-specific and overall survival 
among patients with medullary thyroid cancer. Int. J. Endocrinol. 2020, 8888677 (2020)

23. Y. Ding, Z. Mao, J. Ruan, X. Su, L. Li, T.J. Fahey 3rd, W. Wang, L. Teng, Nomogram-based new recurrence predicting system in early-stage papillary thyroid cancer. Int J. Endocrinol. 2019, 1029092 (2019)

24. V. Ilera, L.C. Delfino, A. Zunino, P. Glikman, M. Drnovsek, A. Reyes, A. Dios, J. Toibaro, V. Pachioli, N. Lannes, A. Guida, A. Gauna, Correlation between inflammatory parameters and pituitary-thyroid axis in patients with COVID-19. Endocrine 74, 455-460 (2021)

25. C. Cox, M. Bosley, L.B. Southerland, S. Ahmadi, J. Perkins, S. Roman, J.A. Sosa, D. Carneiro-Pla, Lobectomy for treatment of differentiated thyroid cancer: can patients avoid postoperative thyroid hormone supplementation and be compliant with the American Thyroid Association guidelines? Surgery 163, 75-80 (2018)

26. Y.M. Lee, M.J. Jeon, W.W. Kim, T.Y. Sung, K.W. Chung, Y.K. Shong, S.J. Hong, Optimal thyrotropin suppression therapy in low-risk thyroid cancer patients after lobectomy. J. Clin. Med. 8, 1279 (2019)

27. Z. Wang, T.E. Angell, W. Sun, Y. Qin, L. He, W. Dong, D. Zhang, T. Zhang, L. Shao, C. Lv, P. Zhang, H. Guan, H. Zhang, Analysis of the strategy of LT4 prescribing and TSH monitoring for thyroid carcinoma after lobectomy. Ann. Transl. Med. 8, 1238 (2020) 